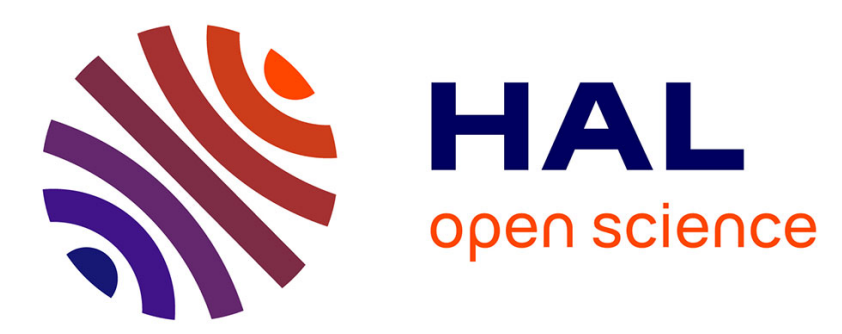

\title{
A structural investigation of the liquid crystal phases of 4-(2'-methylbutyl)phenyl 4'-n-octylbiphenyl-4-carboxylate
}

\author{
J. Budai, R. Pindak, S.C. Davey, J.W. Goodby
}

\section{- To cite this version:}

J. Budai, R. Pindak, S.C. Davey, J.W. Goodby. A structural investigation of the liquid crystal phases of 4-(2'-methylbutyl)phenyl 4'-n-octylbiphenyl-4-carboxylate. Journal de Physique Lettres, 1984, 45 (21), pp.1053-1062. 10.1051/jphyslet:0198400450210105300 . jpa-00232449

HAL Id: jpa-00232449

https://hal.science/jpa-00232449

Submitted on 1 Jan 1984

HAL is a multi-disciplinary open access archive for the deposit and dissemination of scientific research documents, whether they are published or not. The documents may come from teaching and research institutions in France or abroad, or from public or private research centers.
L'archive ouverte pluridisciplinaire HAL, est destinée au dépôt et à la diffusion de documents scientifiques de niveau recherche, publiés ou non, émanant des établissements d'enseignement et de recherche français ou étrangers, des laboratoires publics ou privés. 
Classification

Physics Abstracts

61.30

\title{
A structural investigation of the liquid crystal phases of 4-(2'-methylbutyl)phenyl $4^{\prime}$-n-octylbiphenyl-4-carboxylate
}

\author{
J. Budai, R. Pindak, S. C. Davey and J. W. Goodby \\ AT \& T Bell Laboratories, Murray Hill, New Jersey 07974, U.S.A.
}

(Reçu le 7 mai 1984, révisé le 19 juillet, accepté le 12 septembre 1984)

\begin{abstract}
Résumé. - On a étudié à l'aide de microscopie optique polarisée, miscibilité, DSC et diffraction de rayons $\mathrm{X}$ les phases smectiques du composé 2M4P8BC. On a déterminé la séquence de phases $\mathrm{Cr} \leftrightarrow \mathrm{G} \leftrightarrow \mathrm{J} \leftrightarrow \mathrm{I} \leftrightarrow \mathrm{C} \leftrightarrow \mathrm{A}$ et montré que 2M4P8BC est donc le premier exemple d'un matériau pur avec une transition de phase de $J$ à $G$. Dans sa forme optiquement pure, la phase $I^{*}$ est une phase chirale hexatique alors que les phases $\mathrm{J}^{*}$ et $\mathrm{G}^{*}$ sont des phases cristallines non chirales. On a aussi déterminé les paramètres de réseau de la phase monoclinique $\mathrm{C}$-centrée.
\end{abstract}

\begin{abstract}
The smectic phases of the compound 4-(2'-methylbutyl)phenyl 4'-n-octylbiphenyl4-carboxylate (2M4P8BC) were studied by means of polarized optical microscopy, miscibility, DSC, and X-ray diffraction techniques. The phase sequence $\mathrm{Cr} \leftrightarrow \mathrm{G} \leftrightarrow \mathrm{J} \leftrightarrow \mathrm{I} \leftrightarrow \mathrm{C} \leftrightarrow \mathrm{A}$ has been determined, and consequently $2 \mathrm{M} 4 \mathrm{P} 8 \mathrm{BC}$ has been shown to be the first example of a pure material exhibiting a $J$ to $G$ phase transition. In the optically pure form, the $I^{*}$ phase is a chiral hexatic phase, whereas the $\mathrm{J}^{*}$ and $\mathrm{G}^{*}$ phases were found to be nonchiral crystal phases. The $\mathrm{C}$-centred monoclinic lattice parameters have been determined.
\end{abstract}

\section{Introduction.}

Over recent years, the structural parameters which are commonly associated with separate smectic miscibility groups have been realized [1-3]. One of these structural parameters, the molecular tilt orientation, was shown to be the distinguishing feature between the smectic phases I and $\mathrm{F}[4,5]$. Both phases are commonly considered to be stacked hexatic (short range in-plane positional order with long range bond-orientational order) structures which differ in the direction of the molecular tilt [3]. In the I phase the tilt is towards the apex, while in the F phase it is towards the side of the hexagonal packing array. It should be pointed out, however, that X-ray diffraction studies have shown that the in-plane positional order is quasi-long ranged in the I phase of members of the TBBA series $[5,6]$. Hence, the nature of the positional correlations may differ among compounds currently grouped in the smectic I miscibility class. These miscibility classes will probably evolve as more joint structural and miscibility studies are completed.

A similar situation also applied in the analogous tilted crystal phases. Gane et al. [7], indicated that two different tilt directions were possible. To distinguish between the two, the $G$ phase was defined as the crystal analogue of $F$, and the code letter $G^{\prime}$ was introduced to describe the phase with the tilt towards the apex, i.e., analogous to I. At this point in time however, there was no miscibility evidence to suggest that the two structural forms had separate identities. 
At a later date, Gane et al. [8] resolved this situation by tentatively showing that a transition between the $G^{\prime}$ and $G$ phases could be possible in binary mixtures. On the basis of this evidence, the $\mathrm{G}^{\prime}$ phase was given a new code letter - J. Recently, Diele et al. [9] have described evidence for a $G$ to $J$ phase transition in a binary mixture.

In this present study we have investigated the properties of the material 4-( $2^{\prime}$-methylbutyl) phenyl 4'-n-octylbiphenyl-4-carboxylate (2M4P8BC) in both its racemic and optically active forms. An early study of this material described a tentative phase sequence [10]. A later study described the response of this material to an applied electric field, and suggested the presence of an additional phase [11]. However, the detailed structure of the phases in this material has not yet been elucidated. In the present study, polarized optical microscopy, miscibility, DSC and X-ray structural characterization determined the following phase sequences

$$
\begin{array}{ll} 
\pm 2 \text { M4P8BC } & \text { Iso } \leftrightarrow \mathrm{N} \leftrightarrow \mathrm{A} \leftrightarrow \mathrm{C} \leftrightarrow \mathrm{I} \leftrightarrow \mathrm{J} \leftrightarrow \mathrm{G} \leftrightarrow \text { Cry } \\
+2 \mathrm{M} 4 \mathrm{P} 8 \mathrm{BC} & \text { Iso } \leftrightarrow \mathrm{BP} \leftrightarrow \mathrm{Ch} \leftrightarrow \mathrm{A} \leftrightarrow \mathrm{C}^{*} \leftrightarrow \mathrm{I}^{*} \leftrightarrow \mathrm{J}^{*} \leftrightarrow \mathrm{G}^{*} \leftrightarrow \mathrm{Cry} .
\end{array}
$$

Thus 2M4P8BC is the first example of a pure material exhibiting a $J$ to $G$ phase transition, confirming that the two phases are not of the same miscibility class. Furthermore, unlike the $\mathrm{C}^{*}$ and $\mathrm{I}^{*}$ smectic phases of the optically active material, the $\mathrm{J}^{*}$ and $\mathrm{G}^{*}$ phases do not exhibit a helical arrangement of the tilt director $\left({ }^{1}\right)$. However, unlike their racemic counterparts, the $\mathrm{J}^{*}$ and $\mathrm{G}^{*}$ phases were found to exhibit ferroelectric behaviour $[11,12]$.

\section{Microscopic textural studies.}

Microscopic investigations were carried out on a Zeiss universal polarizing light microscope fitted with a Mettler FP52 microfurnace in conjunction with a FP5 control unit.

The intermediate phase, $J$, and the ensuing $J$ to $G$ phase transition could not be detected from observation of changes in the focal-conic texture. However, the $J$ to $G$ transition is easily detected by examining changes that occur in the schlieren-mosaic patterns of the material. Plates 1 to 4 show the changes which occur on cooling the schlieren texture of the $\mathrm{C}$ phase of the racemate. The $C$ phase exhibits a typical schlieren texture (plate 1), and on cooling it forms a second schlieren texture for the I phase (plate 2). The I to $\mathrm{J}$ transition is characterized by the formation of a mosaic texture which showed some resemblance to the analogous textures of some $F$ phases (plate 3 ). The transition to the $\mathrm{G}$ phase was accomplished via a breathing motion of the mosaic domains, and by the formation of grain boundaries within these domains (plate 4). The $J$ and $G$ phases can be distinguished by comparing plates 3 and 4 . However, this comparison can only be made for a material which exhibits both phases; where they are exhibited singly in different materials, the texture of $\mathbf{J}$ and $\mathbf{G}$ appear indistinguishable.

\section{Miscibility criteria.}

Several miscibility studies were undertaken in order to classify the intermediary phase between I and G. Miscibility studies involving standard materials which exhibit smectic $F$ phases consistently showed similar results. The $F$ and I phases were annihilated in the central portion of the phase diagram and a smectic B phase was injected. A typical miscibility diagram of state for this type of phase behaviour is shown on the right in figure 1 for binary mixtures of 2M4P8BC with terephthalylidene-bis-4-n-nonylaniline (TBNA) [13] as the standard material (A, C, I, F, and $G$ phases). The only phases shown to be continuously miscible were the $A$ and $G$ phases.

( ${ }^{1}$ ) The asterisk is used to denote a phase comprised of optically active molecules in which the reduced environmental symmetry gives rise to ferroelectric properties, and does not necessarily imply a helical structure. 


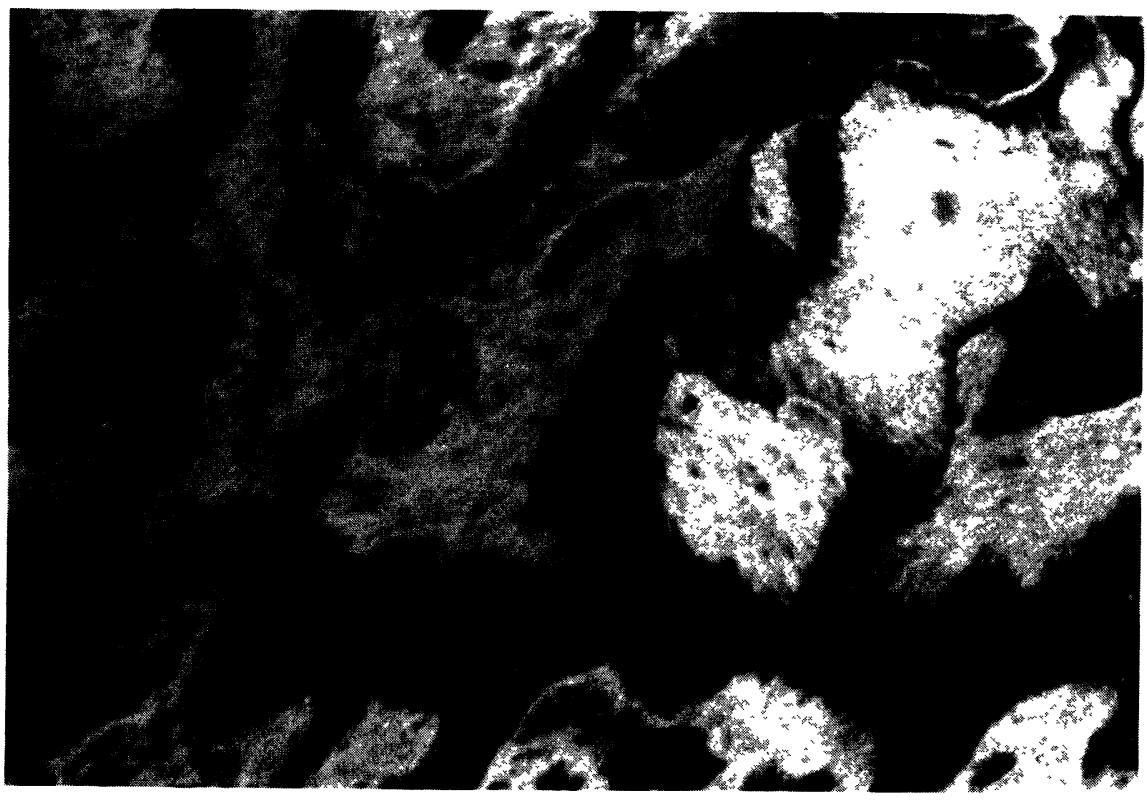

Plate 1. - Schlieren texture of the smectic C phase of $\pm 2 \mathrm{M} 4 \mathrm{P} 8 \mathrm{BC}$.

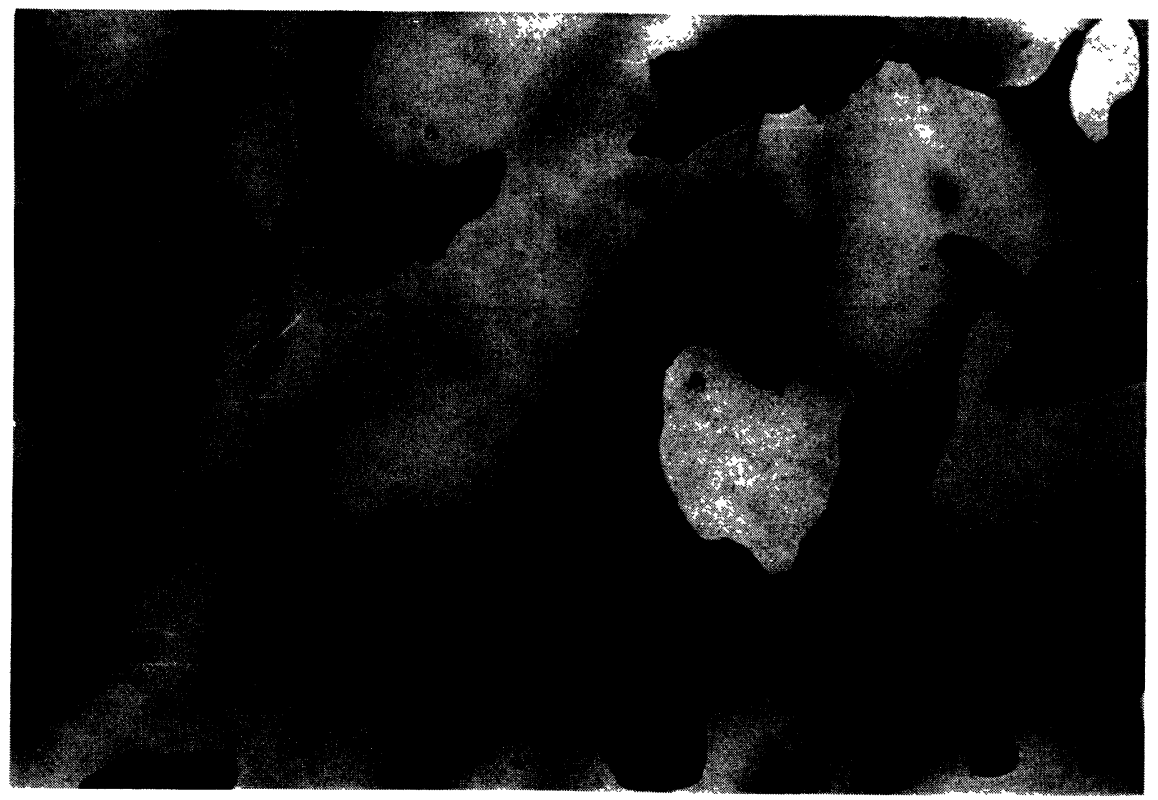

Plate 2. - Schlieren texture of the smectic I phase of \pm 2M4P8BC. 


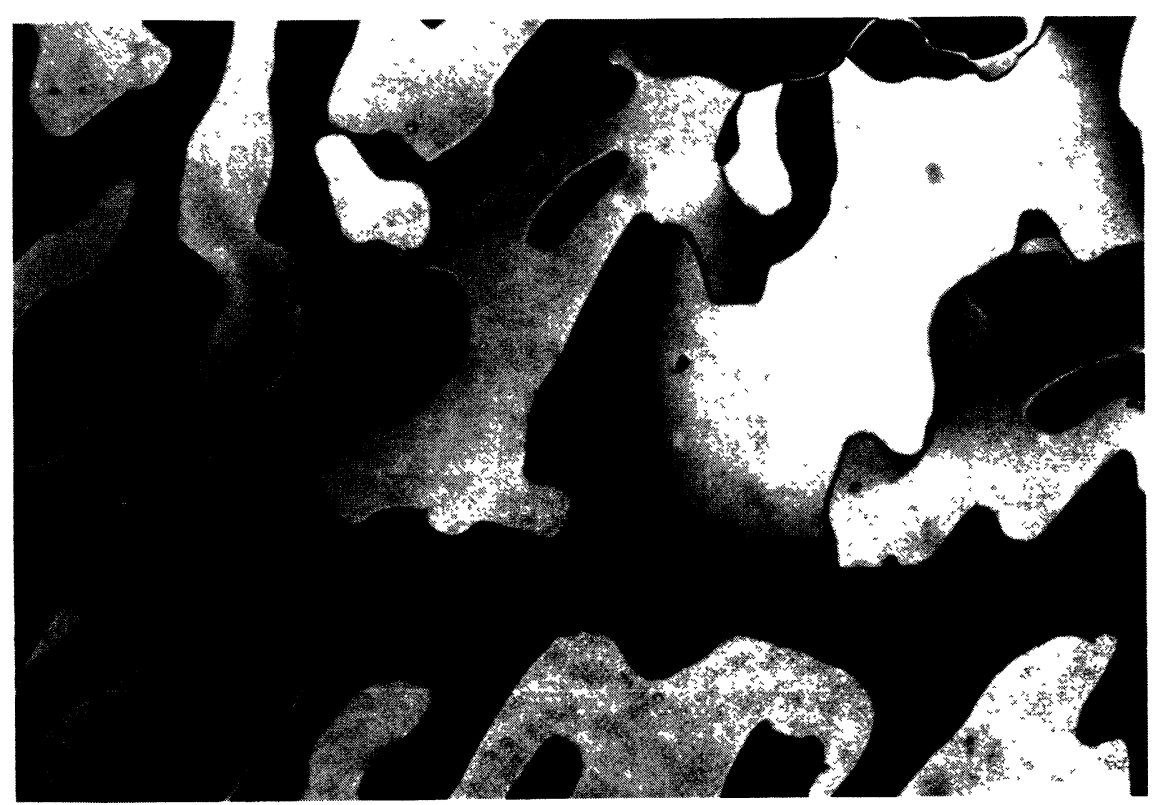

Plate 3. - Schlieren-mosaic texture of the crystal J phase of $\pm 2 \mathrm{M} 4 \mathrm{P} 8 \mathrm{BC}$.

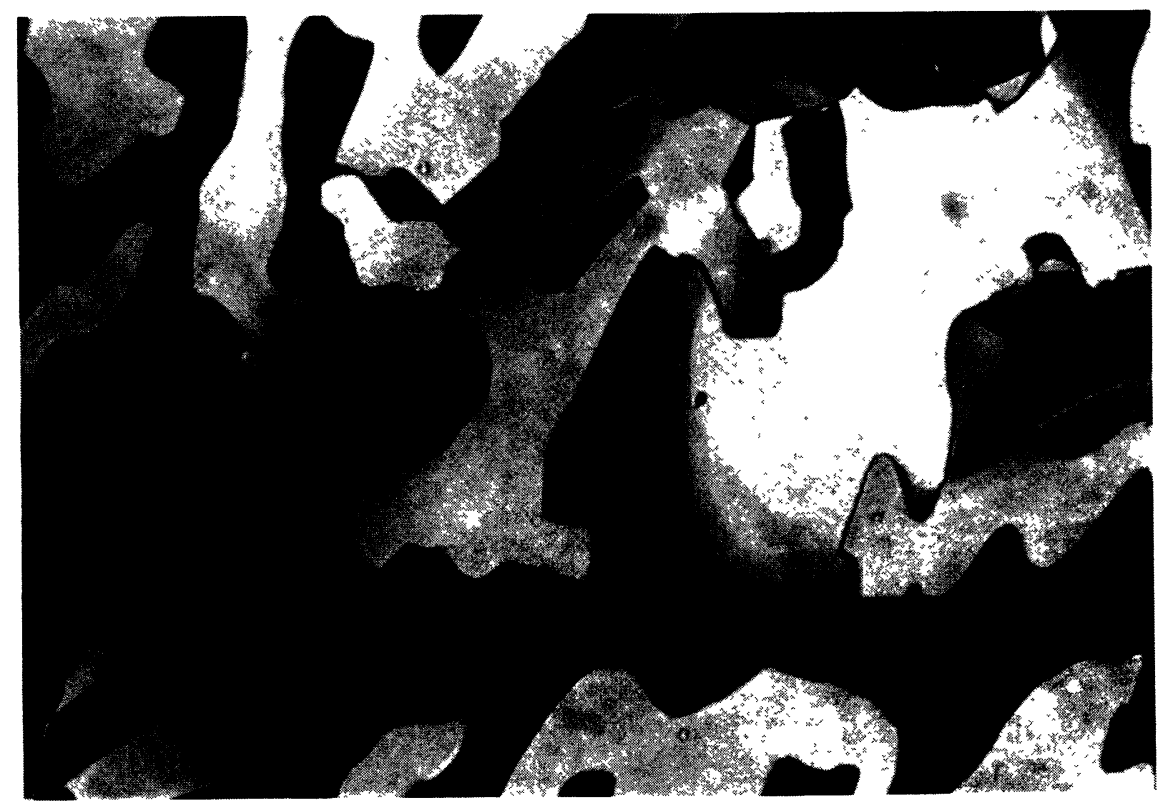

Plate 4. - Mosaic texture of the crystal G phase of $\pm 2 \mathrm{M} 4 \mathrm{P} 8 \mathrm{BC}$. The mosaic domains from the previous phase are further divided by grain boundaries. 


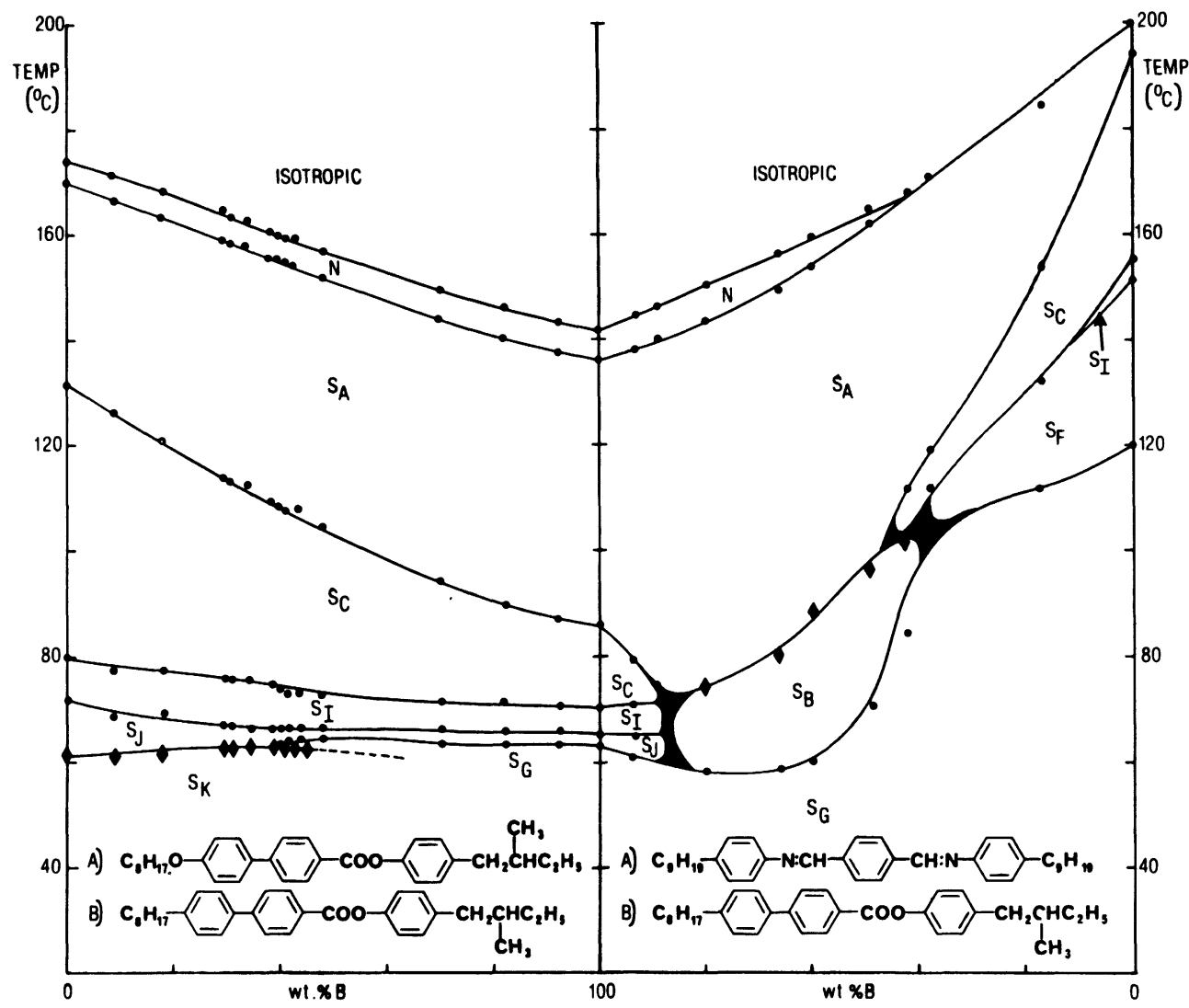

Fig. 1. - Miscibility diagram of state for binary mixtures (by wt. $\%$ ) of $\pm 2 \mathrm{M} 4 \mathrm{P} 8 \mathrm{BC}$ (centre vertical axis) with $\pm 2 \mathrm{M} 4 \mathrm{P} 80 \mathrm{BC}$ (left axis) and TBNA (right axis).

The intermediate phase between $\mathrm{I}$ and $\mathrm{G}$ in 2M4P8BC appeared to be miscible with the $\mathrm{J}$ phase of the standard materials. For example, with the standard compound 4-(-2'-methylbutyl) phenyl 4'-n-octyloxybiphenyl-4-carboxylate (2M4P80BC or 80SI) which exhibits N, A, C, I, $\mathrm{J}$, and $\mathrm{K}$ phases $[4,10,7]$, all of the phases exhibited by $2 \mathrm{M} 4 \mathrm{P} 8 \mathrm{BC}$ appeared to be miscible except for the $\mathrm{G}$ phase. The left side of figure 1 shows the phase diagram for 2M4P80BC and 2M4P8BC. In the $40 \%$ by wt region of $2 \mathrm{M} 4 \mathrm{P} 8 \mathrm{BC}$ a J, G, K phase sequence is observed; however, as the percentage of $2 \mathrm{M} 4 \mathrm{P} 8 \mathrm{BC}$ is increased above $45 \%$ the $\mathrm{K}$ phase abruptly disappears. This may be due to the transition from $\mathbf{G}$ to $\mathbf{K}$ becoming more difficult to observe as the kinetics of the phase transitions become increasing slower at lower temperatures.

Miscibility studies have also been carried out between 2M4P8BC and the compounds HOBACPC and DOBAMBC [14]. Complete miscibility was found in the A, C, and I phases for both compounds. Thus, all of the miscibility results indicate that $2 \mathrm{M} 4 \mathrm{P} 8 \mathrm{BC}$ exhibits a N, A, C, I, J, G phase sequence.

\section{Differential scanning calorimetry.}

Differential scanning calorimetry of 2M4P8BC was performed with a Perkin-Elmer DSC4TADS system fitted with an intracooler. No peaks in the temperature region of the $J$ to $G$ transition were found that could be reliably separated from the background noise, indicating that the transition enthalpy is less than $1-2 \mathrm{~J} \mathrm{~mol}^{-1}$. 
It should be noted that crystalline structure changes which lack a thermal signature have been observed in other liquid crystal systems. Studies of the nO.m series have reported changes within the crystal B phase in which the stacking sequence of the hexagonal layers is altered without any detectable thermal effect $[7,15]$. The entropy change involved in the $J$ to $G$ transition in 2M4P8BC may be comparable to that in restacking transitions in the nO.m series.

\section{X-ray diffraction.}

The structural characterization of the various liquid crystal phases has been carried out by means of X-ray diffraction. Free-standing liquid crystal films were prepared as previously described [16], providing well-aligned specimens containing large $\left(\sim \mathrm{mm}^{2}\right)$ domains. In order to obtain diffraction measurements, a bent graphite monochromator was used to focus the $\mathrm{CuK}_{\alpha}$ $\mathrm{X}$-rays onto the specimen which was mounted on a three-axis Huber diffractometer. The scattered intensity was analysed with a flat graphite crystal and monitored with a scintillation detector. The results which will be presented here were obtained using optically active $+2 \mathrm{M} 4 \mathrm{P} 8 \mathrm{BC}$. The racemic form was found to show similar results.

As mentioned earlier, the crystal phases $\mathrm{J}$ and $\mathrm{G}$ (or $\mathrm{J}^{*}$ and $\mathrm{G}^{*}$ ) differ in their direction of tilt. Both phases have a quasi-hexagonal molecular arrangement within the smectic planes and their lattices can be described using a C-centred monoclinic unit cell. In such a description, the $\mathrm{J}$ phase unit cell tilts towards the larger side of the rectangular basis, while the $\mathrm{G}$ phase tilts towards the smaller.

The corresponding reciprocal lattices for $\mathrm{J}$ and $\mathrm{G}$ structures are shown in figure 2 , with $Q_{\perp}$ and $Q_{\|}$representing the components of the momentum transfer which are perpendicular and parallel respectively to the smectic layers. The angle between the director, $\hat{n}$, and the layer nor-

CRYSTAL $J$

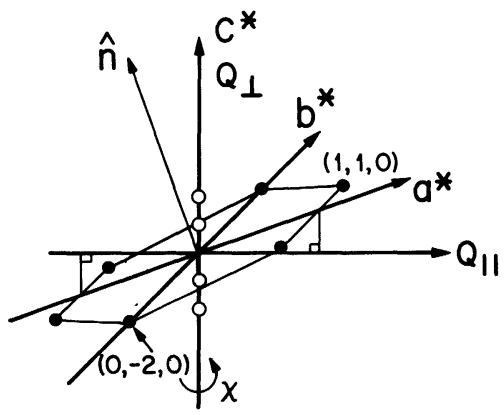

(a)

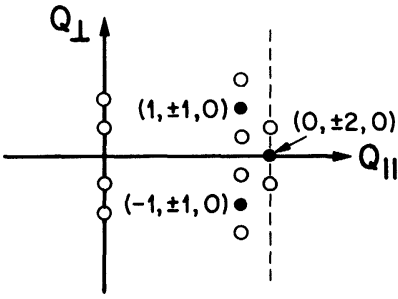

(c)
CRYSTAL G

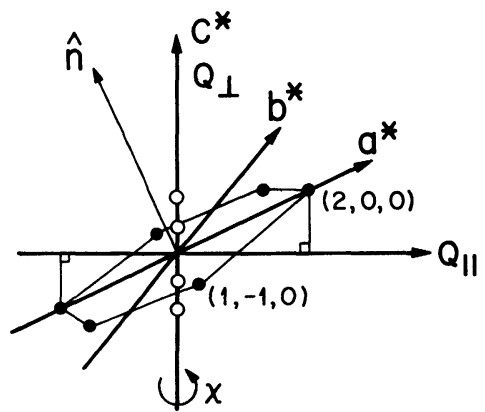

(b)

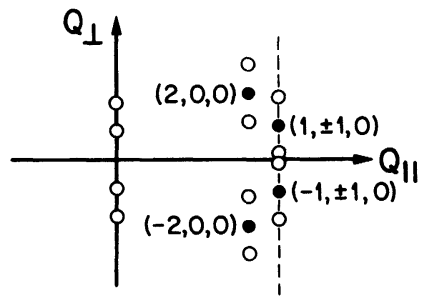

(d)

Fig. 2. - Schematic diagram of the reciprocal lattice for (a) crystal $J$ and (b) crystal $G$ phases. Filled in circles denote $l=0$ reflections. The lower diagrams show a two-dimensional section of the $\chi$-averaged lattice for (c) crystal J and (d) crystal G. 
mal, $c^{*}$, is equal to the molecular tilt. The filled-in circles represent the $l=0$ reflections which result from the quasi-hexagonal in-plane packing. Since the $\mathbf{J}$ and $\mathbf{G}$ phases have a full threedimensional lattice periodicity, $h k l$ reflections with $l \neq 0$ should also be observed and such reflections are shown as open circles (only a few are drawn for clarity).

One distinguishing feature between the reciprocal lattices associated with crystal $\mathrm{J}$ and crystal $\mathrm{G}$ is the presence of $l=0$ Bragg peaks in the $Q_{\perp}=0$ plane in figure 2a and the absence of such peaks in figure $2 b$. This distinction between $J$ and $G$ is maintained even if the reciprocal lattice is rotated about the $c^{*}$ axis ( $\chi$-rotation) which could result from the presence of either a helical molecular arrangement or a mosaic domain structure in the sample. The intersection of rotated $J$ and $\mathrm{G}$ reciprocal lattices with a $Q_{\perp}, Q_{\|}$plane is shown in figures $2 \mathrm{c}, \mathrm{d}$.

The local molecular arrangement for the hexatic $\mathrm{F}$ and $\mathrm{I}$ phases is analogous to that for the crystal $G$ and $J$ phases. Thus the hexatic phases can be described in terms of the same $C$-centred monoclinic unit cell which has been described above. However, the loss of interlayer correlations for the hexatic phases results in continuous rods of scattering, rather than Bragg peaks, parallel to $Q_{\perp}$. In addition, the short-range, in-plane positional order of the hexatic phases should give rise to a Lorentzian structure factor given by

$$
S\left(\mathbf{Q}_{\|}\right) \sim\left[\xi_{\|}^{2}\left(\mathbf{Q}_{\|}-\mathbf{Q}_{0}\right)^{2}+1\right]^{-1}
$$

where $\xi_{\|}$is the in-plane positional correlation length and $\mathbf{Q}_{0}$ is the lowest order reciprocal lattice vector.

5.1 OBSERVATIONS. - The peak diffracted intensity measured as a function of $Q_{\perp}$ is shown in figure $3 a$ for four different temperatures. Bragg peaks with $l \neq 0$ are observed for the $G^{*}$ and $\mathrm{J}^{*}$ phases, verifying the existence of long-range interlayer correlations. In the $\mathrm{I}^{*}, \mathrm{C}^{*}$, and A phases a relatively weak continuous rod of scattering shows that the interlayer correlation length is limited to approximately one layer. Figure $3 \mathrm{~b}$ shows the measured X-ray intensity as a function of $Q_{\|}$for the same four temperatures. The width of the peaks in the $\mathrm{G}^{*}$ and $\mathrm{J}^{*}$ phases is equal to the instrumental resolution width (FWHM $\sim 0.013 \AA^{-1}$ ), whereas the width in the $I^{*}$ and $\mathrm{A}$ phases is significantly larger.

$\mathrm{X}$-ray scans taken while rotating the specimen about the layer normal ( $\chi$ scans) were also obtained. In the smectic A phase, the peak intensity as a function of the azimuthal angle was constant, as expected for liquid-like in-plane order. In the $I^{*}$ phase, the measured intensity showed only a small modulation over a $60^{\circ}$ range in $\chi$. This observation is consistent with the presence of long-range bond-orientational order which is largely averaged out by the helical molecular arrangement. The $\chi$ scans through the peaks in the $\mathrm{G}^{*}$ and $\mathrm{J}^{*}$ phases had a narrow width approaching that of the instrumental resolution, showing that the helicity has been suppressed. For our film thickness of $\sim 1 \mu \mathrm{m}$, a lower limit of approximately $100 \mu \mathrm{m}$ can be set for the value of the helical pitch in the crystal phases (if any exists at all).

5.2 LATTICE PARAMETERS. - Using X-ray intensity scans to map out the reciprocal lattice for each phase, the C-centred monoclinic unit cell lattice parameters were obtained

$$
\begin{array}{lllll}
\mathrm{G}^{*} & a=9.60 \AA & b=5.3 \AA & c=30.8 \AA & \beta=109.5^{\circ} \\
\mathrm{J}^{*} & a=5.6 \AA & b=9.16 \AA & c=30.8 \AA & \beta=1090 \\
\mathrm{I}^{*} & a=5.5 \AA & b=9.15 \AA & & \beta=108^{\circ} .
\end{array}
$$

The hexagonal arrangement within a smectic layer is clearly distorted in all three tilted phases. However, this distortion is not surprising since a plane lying perpendicular to the molecular tilt axes shows a regular hexagonal lattice, as it would for a close packed array of tilted cylinders. The tilt angle between the molecular axes and the layer normal is given by $\beta-90^{\circ}$ and decreases with increasing temperature. 


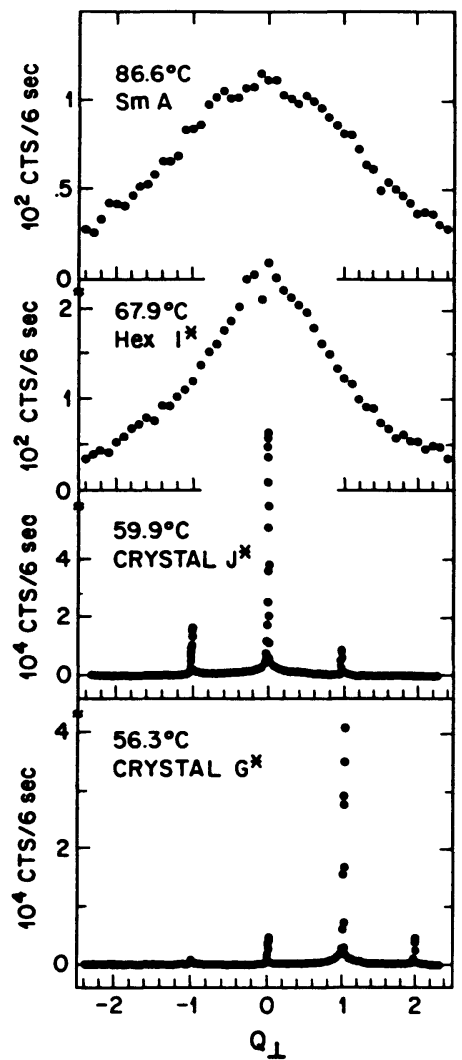

(a)

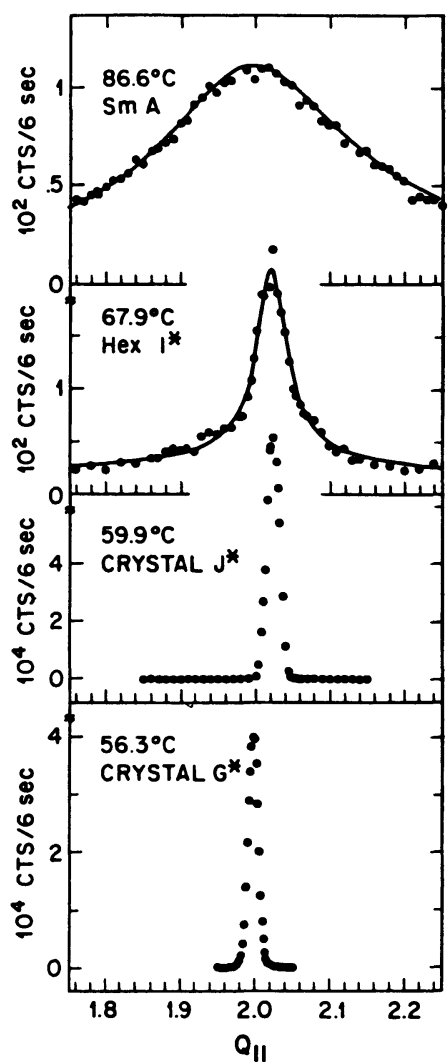

(b)

Fig. 3. - (a) Intensity through reciprocal lattice peaks as a function of the out-of-plane momentum transfer, $Q_{\perp}$, in units of $0.216 \AA^{-1}$. (b) Intensity through the $l=0$ peaks as a function of the in-plane momentum, $Q_{\|}$, in units of $0.677 \AA^{-1}$.

5.3 Discussion. - The solid lines which are shown in figure $3 b$ represent the least squares fit between the intensity data and a Lorentzian structure factor. In obtaining these curves, the $\chi$-integrated form of equation (1) was convoluted with the Gaussian instrumental resolution function. The fits yield values for the in-plane correlation length, $\xi_{\|}$, of $120 \AA$ at $67.9^{\circ} \mathrm{C}\left(\mathrm{Hex} \mathrm{I} \mathrm{I}^{*}\right)$ and $12 \AA$ at $86.6^{\circ} \mathrm{C}(\mathrm{SmA})$. Within the $\mathrm{I}^{*}$ phase, $\xi_{\|}$was observed to increase with decreasing temperature and was approximately $180 \AA$ at $61.1^{\circ} \mathrm{C}$.

In X-ray diffraction studies of the I phase in the terephthalylidene-bis-4-n-alkylanilines (TBAA) compounds, Benattar et al. $[5,6]$ found that the experimental intensity lineshapes could not be fitted by a Lorentzian structure factor. Instead, a power law dependence corresponding to a stacked two-dimensional crystal was required. We have attempted to fit our data for $+2 \mathrm{M} 4 \mathrm{P} 8 \mathrm{BC}$ in the I* phase to the $\chi$-integrated form of the structure factor $\left|\mathbf{Q}_{\|}-\mathbf{Q}_{0}\right|^{-2+\eta}$. A reasonable fit could not be obtained for any value of $\eta$ less than $1 / 2$. Since the maximum value of $\eta$ predicted by theory [17] is $1 / 3$, we conclude that the $I^{*}$ phase in $+2 \mathrm{M} 4 \mathrm{P} 8 \mathrm{BC}$ is a hexatic rather than a two-dimensional crystal. The apparent difference in the range of the positional order in $+2 \mathrm{M} 4 \mathrm{P} 8 \mathrm{BC}$ and TBDA, together with the observation from figure 1 that the I phases for 2M4P8BC and TBNA are not continuously miscible suggest that two structurally different I phases may exist. 
To the left of the $S_{B}$ region of the phase diagram, the phase sequence is given by $S_{C}-S_{1}-S_{\text {J }}$, whereas on the right side the sequence is $S_{C}-S_{F}-S_{G}$. In the $S_{C}$ phase, it has been theoretically shown that the molecular tilt should induce long-ranged bond-orientational order [18], and thus there is no fundamental distinction between $S_{C}$ and a tilted hexatic phase. This induced bondorientational order has never been observed experimentally in the $S_{C}$ phase. Nevertheless, since the $S_{C}$ phases of 2M4P8BC and TBNA do not show complete miscibility and, near the centre of the phase diagram, lie above phases which exhibit different tilt directions with respect to the six-fold symmetric bond-order, this miscibility behaviour suggests the existence of two structurally distinct $S_{C}$ phases which also exhibit different tilt directions with respect to their six-fold symmetric bond-order [19]. Of course, this miscibility evidence is indirect and further structural evidence would be required to first confirm the existence of bond-orientational order in the $S_{C}$ phase and then to study the effect of such order on phase diagrams.

While obtaining the X-ray measurements, several important characteristics of free standing films of +2 M4P8BC were noted. First, while rotating the film about its normal, the $\chi$ value of a Bragg peak for a particular domain was sometimes observed to change slowly $(\sim 1 \% / \mathrm{min})$ in time. These changes in $\chi$ were commonly observed in the $\mathrm{J}^{*}$ and $\mathrm{I}^{*}$ phases, and only very rarely in the $G^{*}$ phase. This rotation could be due either to the rotation of the molecular tilt direction within a domain among the six equivalent hexagonal directions or to the spatial migration or rotation of the domain. Second, the kinetics associated with the $G^{*} \leftrightarrow J^{*} \leftrightarrow I^{*}$ phase transitions can proceed extremely slowly near the transition temperatures. Often many hours of equilibration were required before a phase would fully establish itself. This time dependence made it difficult to accurately determine the transition temperatures and together with sample differences, may explain why the $\pm 2 \mathrm{M} 4 \mathrm{P} 8 \mathrm{BC}$ transition temperatures detected by textural changes (scan rate $\sim 1{ }^{\circ} \mathrm{C} / \mathrm{min}$ ) of $\mathrm{G} \stackrel{63^{\circ}}{\longleftrightarrow} \mathrm{J} \stackrel{65^{\circ}}{\longleftrightarrow}$ I were slightly elevated when compared with the +2 M4P8BC X-ray results of $\mathrm{G}^{*} \stackrel{59^{\circ} \mathrm{C}}{\longrightarrow} \mathrm{J}^{*} \stackrel{61^{\circ} \mathrm{C}}{\longrightarrow} \mathrm{I}^{*}$. It should be noted however, that the two samples of $\pm 2 \mathrm{M} 4 \mathrm{P} 8 \mathrm{BC}$ and $+2 \mathrm{M} 4 \mathrm{P} 8 \mathrm{BC}$ were of comparable purity, in excess of $99 \%$ by HPLC analysis.

Finally, it was noted that free standing films of $2 \mathrm{M} 4 \mathrm{P} 8 \mathrm{BC}$ as thin as two-molecular layers can be prepared. Thus, future studies in which the film thickness is reduced should provide useful information on the evolution from 3-dimensional order to 2-dimensional order in a system where the tilt direction can couple to the bond orientational order.

\section{References}

[1] De VRIES, A., J. Chem. Phys. 70 (1979) 2705.

[2] De VRies, A., Mol. Cryst. Liq. Cryst. 63 (1981) 215.

[3] Goodby, J. W., Mol. Cryst. Liq. Cryst. Lett. 92 (1983) 171.

[4] Leadbetter, A. J., Gaughan, J. P., Kelly, B., Gray, G. W. and Goodby, J. W., J. Physique Colloq. 40 (1979) C3-178.

[5] Benattar, J. J., Moussa, F., Lambert, M. and Germain, C., J. Physique Lett. 42 (1981) L-67.

[6] Benattar, J. J., Moussa, F. and Lambert, M., J. Chim. Phys. 80 (1983) 99.

[7] Gane, P. A. C., Leadbetter, A. J. and Wrighton, P. G., Mol. Cryst. Liq. Cryst. 66 (1981) 247.

[8] Gane, P. A. C., Leadbetter, A. J., Wrighton, P. G., Goodby, J. W., Gray, G. W. and Tajbakhsh, Mol. Cryst. Liq. Cryst. 100 (1983) 67.

[9] Diele, S., Mädicke, A., Höft, E.-M., Wende, G. and Sackmann, H., Mol. Cryst. Liq. Cryst. Lett. 92 (1983) 187.

[10] Goodby, J. W. and Gray, G. W., J. Physique Colloq. 40 (1979) C3-27.

[11] Brand, H. R. and Cladis, P. E., J. Physique Lett. 45 (1984) L-217.

[12] Patel, J. S., to be published. 
[13] Richter, L., Demus, D. and Sackmann, H., Mol. Cryst. Liq. Cryst. 71 (1981) 269.

[14] Goodby, J. W., unpublished work.

[15] Blcemen, E. and Garland, C. W., J. Physique 42 (1981) 1299.

[16] Moncton, D. E. and Pindak, R., Phys. Rev. Lett. 43 (1979) 701.

[17] Nelson, D. R. and Halperin, B. I., Phys. Rev. B 19 (1979) 2457.

[18] Nelson, D. R. and Halperin, B. I., Phys. Rev. B 21 (1980) 5312.

[19] This was independently suggested to us by A. Saupe and S. Kumar. 